\title{
Differential expression of viral pathogen- associated molecular pattern receptors mRNA in Egyptian chronic hepatitis C virus patients
}

Reda A. Suef ${ }^{1}$, Ezz Elden M. Mohamed ${ }^{1}$, Mohamed T. M. Mansour ${ }^{2}$, Kilian Weigand ${ }^{3}$ and Mohamed M. S. Farag ${ }^{\text {** }^{*}}$

\begin{abstract}
Background: One possible mechanism utilized by hepatitis $C$ virus (HCV) to escape from the host's innate immune surveillance is modification of its pathogen-associated molecular patterns (PAMPs) by altering or hiding its RNA which interfering with toll-like receptors (TLRs) signaling and ultimately hindering the production of proinflammatory cytokines, chemokines, and interferons (IFNs). This study aimed to examine the expression levels of TLR3, TLR7, and IFN-a to investigate the correlated expression pattern among them in chronic HCV patients. Patients included in this study were categorized into two different groups, non-treated chronic HCV patients and treated chronic HCV patients, in addition to healthy volunteers as a control group. The blood samples were assessed for HCVAb, HCVRNA, HCV genotypes, and different biochemical analyses. The mRNA levels of TLR3, TLR7, and IFN-a in peripheral blood of chronic HCV patients were quantitatively measured in comparison to healthy controls.

Results: The expression levels of TLR3, TLR7, and IFN-a were significantly downregulated in non-treated chronic HCV patients compared to both treated HCV patients and control subjects. On the other hand, treated HCV patients showed non-significant downregulation of the same three sensing receptors (TLR3, TLR7, and IFN-a) compared to control group. Obviously, the expression levels of IFN-a were positively correlated with the levels of both TLR3 and TLR7.

Conclusion: The exhausted innate immunity against HCV may correlate to HCV downregulation of TLR3 and TLR7 expression on innate immune cells with a subsequent decrease in INF-a production and the possibility of targeting these receptors to enhance the immune response and clear the infection needs further studies.
\end{abstract}

Keywords: Hepatitis C virus, TLR3 and TLR7 signaling, IFN-a transcription

\section{Background}

$\mathrm{HCV}$ is an enveloped, positive-sense single-stranded RNA virus (ssRNA). It is a member of the Hepacivirus genus within the family Flaviviridae [1]. HCV is grouped into seven major genotypes that display at least 30\% variation in nucleotide sequence. This genetic variation

\footnotetext{
* Correspondence: mohamed.farag@azhar.edu.eg

${ }^{1}$ Botany and Microbiology Department, Faculty of Science, Al-Azhar University, 11884 Nasr City, Cairo, Egypt

Full list of author information is available at the end of the article
}

is responsible for resistance to antiviral drugs and helps in escaping of the immune system [2]. The most common genotype of $\mathrm{HCV}$ in Egypt is genotype 4 with subtype (4a), representing at least $85 \%$ of all $\mathrm{HCV}$ cases in Egypt [3].

The infection with HCV occurs in two phases, acute infection phase that occurs within the first 6 months of the infection and if untreated then $\mathrm{HCV}$ will progress to persistent infection [4]. Acute infection control depends on host- and viral-related factors, the former includes an 
early induction of innate and adaptive immunity. Such responses, however, are insufficient to clear the virus in most cases, typically leads to chronic infection which can be attributed to the virus's escape nature to prevent the host immune response and to resist the IFN antiviral therapy [5].

On HCV, the sensing of the specific ligand by the innate immune receptor triggers a variety of intracellular down streaming signaling cascades that finally acquire the infected and neighboring cells an antiviral immunization state [6]. This antiviral innate immunity depends on different pattern recognition receptors (PRRs) which recognize microbial PAMPs that induce innate immunity following infection [7]. TLRs are a family of membrane-bound PRRs acting as a vital regulator of both innate and adaptive immune response to a wide variety of microbial PAMPs, e.g., lipids, lipoproteins, proteins, nucleic acids, and glycans [8]. To date, 11 kinds of human TLRs have been described [9]. Among them, TLR3, TLR7, and TLR8 are associated with recognition of viral RNA. TLR3 is expressed on dendritic cells (DCs) [10] and activated by double-stranded (dsRNA) that is produced during HCV replication [11]. While TLR7 (expressed by plasmacytoid DCs and B cells) and TLR8 (expressed on monocytes) are recognize HCV ssRNA [12]. Induction of TLR3 by dsRNA can stimulate type I IFN and proinflammatory cytokine production upon activation mediated by interferon regulatory factor 3 (IRF3) and nuclear factor NF-kappa-B (NFkB) based on the adaptor protein TIR-domain-containing adapterinducing interferon- $\beta$ (TRIF) [13]. On the other hand, induction of TLR7 by HCV ssRNA stimulate type I IFN production upon activation mediated by IRF-7 based on the adaptor protein myeloid differentiation primary response 88 (MyD88) [14].

As one of the immune evading strategies that may lead to viral persistence, $\mathrm{HCV}$ can interfere with TLR3 and TLR7 signaling either by blocking their downstream cascades or by interfering with transcription factors itself. Consequently, the downstream mechanism for induction of IFN and proinflammatory cytokines is avoided [15].

For more investigations, the main goal of the present study was therefore to assess the expression of mRNA levels for TLR3 and TLR7 and correlate their expression with the IFN- $\alpha$ production in chronic HCV patients.

\section{Methods}

\section{Patient recruitment procedures}

Patients included in our study were categorized into two different groups: non-treated chronic HCV patients $(n=$ $20)$ and treated HCV patients $(n=10)$, in addition to healthy volunteers $(n=10)$ as a control group. For chronic hepatitis $\mathrm{C}(\mathrm{CHC})$ patients, the diagnosis of $\mathrm{CHC}$ was confirmed by the detection of $\mathrm{HCV}$ RNA using real-time PCR (RT-PCR). Different biochemical markers including ALT, AST, WBCs, HB, and PLT were detected and measured using a routine laboratory method. CHC patients who concurrent with HBV, HDV, or HIV infection, or any immunological disorders were excluded. The treated HCV group was once-weekly injected for 12-18 weeks with interferon-ribavirin (INFRib) combination therapy and the patients were recruited within 6 months after completion of treatment based on sustained virological response (SVR). For controls, participants were negative to $\mathrm{HBV}, \mathrm{HCV}$, and HIV and free from any liver diseases. Control and $\mathrm{CHC}$ subjects were age/sex-matched.

All participants were consented to use their samples and clinical data ( $\mathrm{CHC}$ patients) for this study. Informed written consent was obtained from each patient included in the study. The study protocol was approved by the ethics committee of the National Liver Institute, and the research was conducted in accordance with the principles of the Declaration of Helsinki.

Peripheral blood was collected from HCV patients and healthy donors under sterile and aseptic conditions according to infection control ethics. Peripheral blood mononuclear cells (PBMCs) were collected in PAXgene ${ }^{\mathrm{m}}$ blood RNA tube (PreAnalytix GmbH, Switzerland) which contains an exclusive reagent that stabilizes and inhibits degradation of intracellular RNA molecules by RNases and minimizes induction of gene expression.

\section{Total RNA extraction and cDNA synthesis}

The PAXgene RNA purification kit (Qiagen $\mathrm{GmbH}$, Germany) was used for total RNA extraction and mRNA purified with on-column, DNase set (Qiagen). According to the manufacturer's guidelines, the quantity and purity of propagated RNA were evaluated by measuring absorbance at $260 \mathrm{~nm}$ and the ratio A260/A280 in a UV spectrophotometer (Nanodrop Inc., Wilmington, DE, USA). Extracted RNA that has an A 260/280 value $\geq 2.0$ is considered relatively free of protein and considered valid for RT-qPCR.

For TLRs, mRNA quantification by RT-qPCR, "twostep" RT-qPCR method was used. Firstly, 500 ng of total RNA per sample was transcribed into cDNA using the high-capacity cDNA reverse transcription kit (Applied Biosystems, USA, and Insert P/N 4375222 REV B) with random primers for transcription of RNA into cDNA using reverse transcriptase. Then, $2 \mu \mathrm{l}$ aliquot of the resulting cDNA was used as a template source for amplification RT-qPCR reactions.

\section{Real-time PCR}

RT-qPCR amplification for TLR3, TLR7, and INF- $\alpha$ genes were carried out by Quantistudio 6 flex machine (Applied Biosystems) and achieved by using Maxima 
SYBR Green qPCR Master Mix kit after addition of primers and template according to manufacturer's guidelines. Also, a stable glyceraldehyde 3 phosphate dehydrogenase (GAPDH) used as reference gene. For TLR3, the forward primer was TGGTTGGGCC ACCTAGAAGTA and reverse primer: TCTCCATTCC TGGCCTGTG. For TLR7, the forward primer was TTTACCTGGATGGAAACCAGCTA and the reverse primer was TCAAGGCTGAGAAGCT GTAAGCTA. Regarding IFN $\alpha$, the forward primer was TGCTTTAC TGATGGTCCTGGT and reverse primer was TCATGT CTGTCCATCAGACAG. The GAPDH-forward primer was ATGGCTATGATGGAGGTCCAG and GAPDH-reverse primer was TTGTCCTGCATCTGCTTCAGC [10].

After amplification, melting-curve analysis was performed and PCR reaction specificity confirmed without appearance for contamination or primer-dimer. For the expression analysis of the three target genes (TLR3, TLR, and INF- $\alpha$ ) in tested samples and calibrator using a GAPDH reference gene as the normalizer, comparative (Livak) $2^{-\triangle \Delta C T}$ quantification method was chosen to calculate changes in gene expression where the genes of interest in non-treated group was compared to the same genes in the control group after normalizing the expression to the expression of a stable reference gene in both control and tested samples. The results were expressed as a relative fold up- or downregulation between a test and control.

\section{HCV genotype}

The HCV genotype 2.0 (Inno-LiPA) assay was used for determination of the genotype of $\mathrm{HCV}$. The assay was depending on genotype-specific oligonucleotides at $5^{\prime}$ UTR position that was immobilized on a nitrocellulose strip. The HCV 5' UTR core region was amplified by PCR, and the oligonucleotides were annealed with a biotin-labeled 5' UTR amplicon. The labeled amplicon could pair and be mounted on a strip. After washing, the hybridized products were traced using alkaline phosphatase labeled streptavidin, and BCIP/NBT chromogen was used as a substrate according to the manufacturer's guidelines. The genotype bands were interpreted using the chart supplied by the manufacturer.

\section{Statistical analysis}

Data was analyzed using SPSS v.25 and Sigmaplot v.12.5. Independent sample $t$ test was applied for pairwise comparisons among the groups within each marker. Data representation was done using Excel 365. Grubbs's test (extreme studentized deviate test) was performed to determine whether values in each group is a significant outlier at alpha $=0.05$. At each group, no more than one of the values is furthest from the rest, but not detected as a single significant outlier $(p>0.05)$. Pearson correlation was calculated among different markers within each group, and SigmaPlot 12.5 was used to produce correlation graphs.

\section{Results \\ Descriptive analysis for patients' age}

Patients' age presented by a box plot constructed in the SigmaPlot ${ }^{\circ} 12.5$ software. In the presented study, the age was roughly symmetric around the median of nontreated chronic HCV patients (35 years) with a minimum and maximum range of 21 to 64 years and treated $\mathrm{HCV}$ patients ( 35 years) with a minimum and maximum range of 26 to 57 year (Fig. 1).

\section{Virological findings}

All non-treated chronic HCV patients included in our study were seropositive for anti-HCV antibody and positive for HCV RNA which was detected by RT-PCR. Depending upon the viral load, non-treated chronic $\mathrm{HCV}$ patients were classified into four categories: $1 / 20(5 \%)$ was very low, $8 / 20(40 \%)$ was low, $10 / 20(50 \%)$ was intermediate, and 1/20 (5\%) was high virus titers (Fig. 2). For HCV genotyping, our results showed that all samples were $\mathrm{HCV}$ genotype 4 subtype $4 \mathrm{a}$ except one sample was HCV genotype 4 subtype 4c.

Gene expression of TLR3, TLR7, and IFN-a in study groups In the present study, we have used quantitative RT-PCR to evaluate the expression of PAMP receptors related with the recognition of viral RNA in blood samples. The mRNA relative expression values for IFN- $\alpha$, TLR3, and TLR7 were detected. GAPDH was chosen as a reference

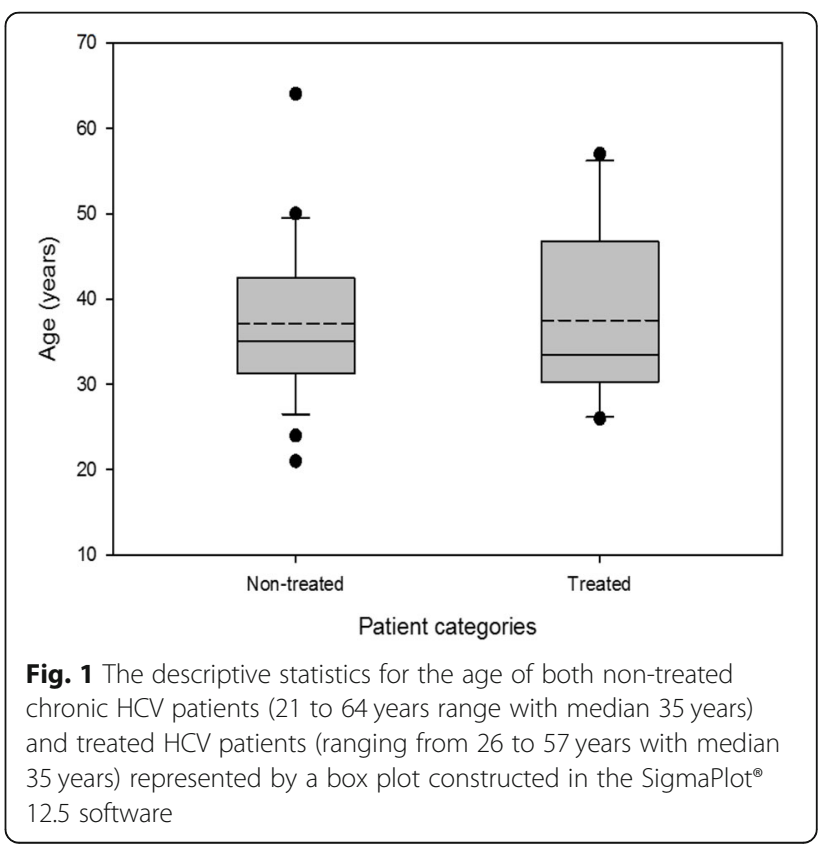




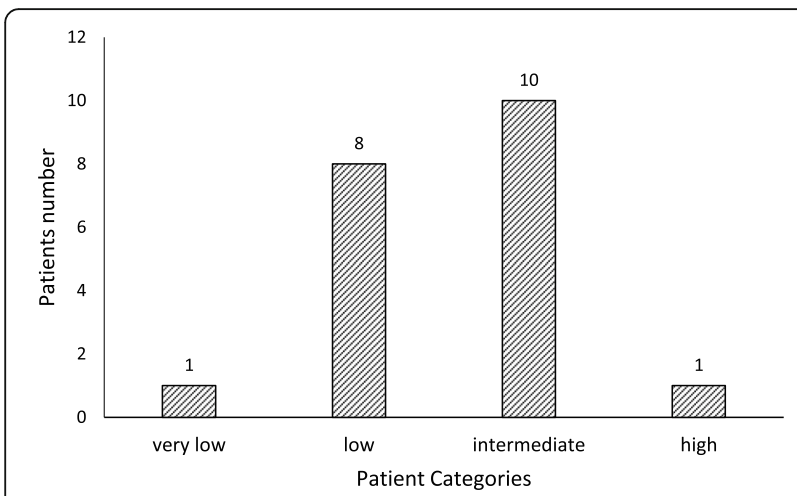

Fig. 2 The patient's classification according to RT-PCR values and virus titers. Very low $\leq 10,000$; low 10,000:100,000; intermediate 100,000:1,000,000; High $\geq 1,000,000$

gene that represents a baseline for the expression of given target genes.

Our results showed that the expression levels of TLR3, TLR7, and IFN- $\alpha$ were significantly lower $(p<0.001)$ in non-treated chronic patients when compared with the control group (Fig. 3). On the other hand, the expression levels of TLR3, TLR7, and IFN- $\alpha$ were non-significant in the treated HCV group when compared with control subjects (Fig. 3). Our results also showed the expression levels of TLR3, TLR7, and IFN- $\alpha$ were significantly lower $(p<0.001)$ in non-treated chronic patients when compared with the treated HCV group (Fig. 3).

\section{Correlation analysis among TLR3, TLR7, and IFN- $a$ genes expression}

In the presented study, the bivariate analysis showed a positive correlation between the expression levels of TLR3 and IFN- $\alpha$ in non-treated chronic HCV patients $(r$
$=0.295$; slope $=0.173 ; p=0.206)$ (Fig. 4a), treated HCV group $(r=0.295$; slope $=0.433 ; p=0.441)$ (Fig. 4b), and control group $(r=0.470$; slope $=0.306 ; p=0.170)$ (Fig. 4c).

On the other hand, the bivariate analysis showed a positive correlation between the expression levels of TLR7 and IFN- $\alpha$ in non-treated chronic HCV patients ( $r$ $=0.267$; slope $=0.367 ; p=0.254$ ) (Fig. 5a), treated HCV group ( $r=0.219$; slope $=0.193 ; p=0.572$ ) (Fig. $5 \mathrm{~b})$, and control group $(r=0.570$; slope $=0.575 ; p=0.086$ ) (Fig. 5c).

Regarding the correlation between the expression levels of TLR3 and TLR7, the bivariate analysis showed a highly significantly positive correlation between the expression levels of TLR3 and TLR7 in non-treated chronic HCV patients $(r=0.567$; slope $=0.236 ; p=$ 0.009) (Fig. 6a), and in control group ( $r=0.974$; slope $=$ $0.629 ; p=0.001$ ) (Fig. 6c). Finally, the bivariate analysis showed a positive correlation between the expression levels of TLR3 and TLR7 in the treated HCV group ( $r=$ 0.381 ; slope $=0.643 ; p=0.312$ ) (Fig. 6b).

\section{Correlation between TLR3, TLR7, IFN- $a$, and biochemical parameters}

The correlation among TLR3, TLR7, IFN- $\alpha$, and all biochemical parameters findings (ALT, AST, WBCs, HB, PLT) in the non-treated chronic HCV patients were non-significant (NS). Regards to HCV RNA viral load the correlation was significant $p<0.001, p=0.004, p<$ 0.001 for TLR3, TLR7, and IFN- $\alpha$, respectively (Table 1 ).

\section{Discussion}

The host's ability to respond to invasive pathogens depends on the activation of the innate immune system

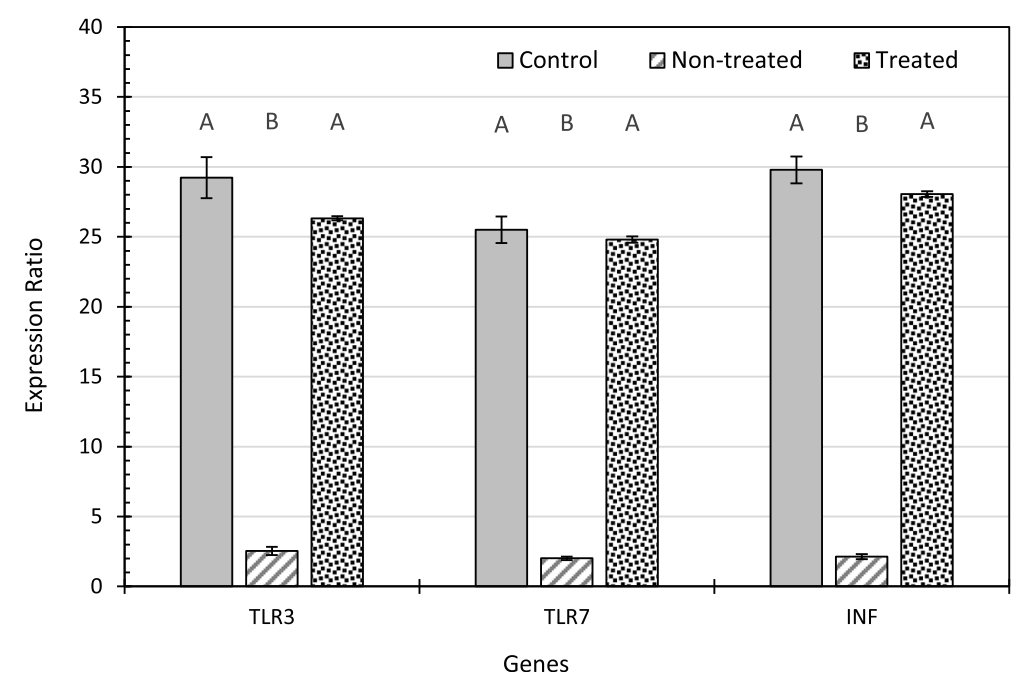

Fig. 3 The comparison of TLR3, TLR7, and IFN-a expression level in non-treated chronic HCV patients, treated HCV patients, and control subjects. Capital letters represent the significance among the groups. Error bars represent standard error (SE) 

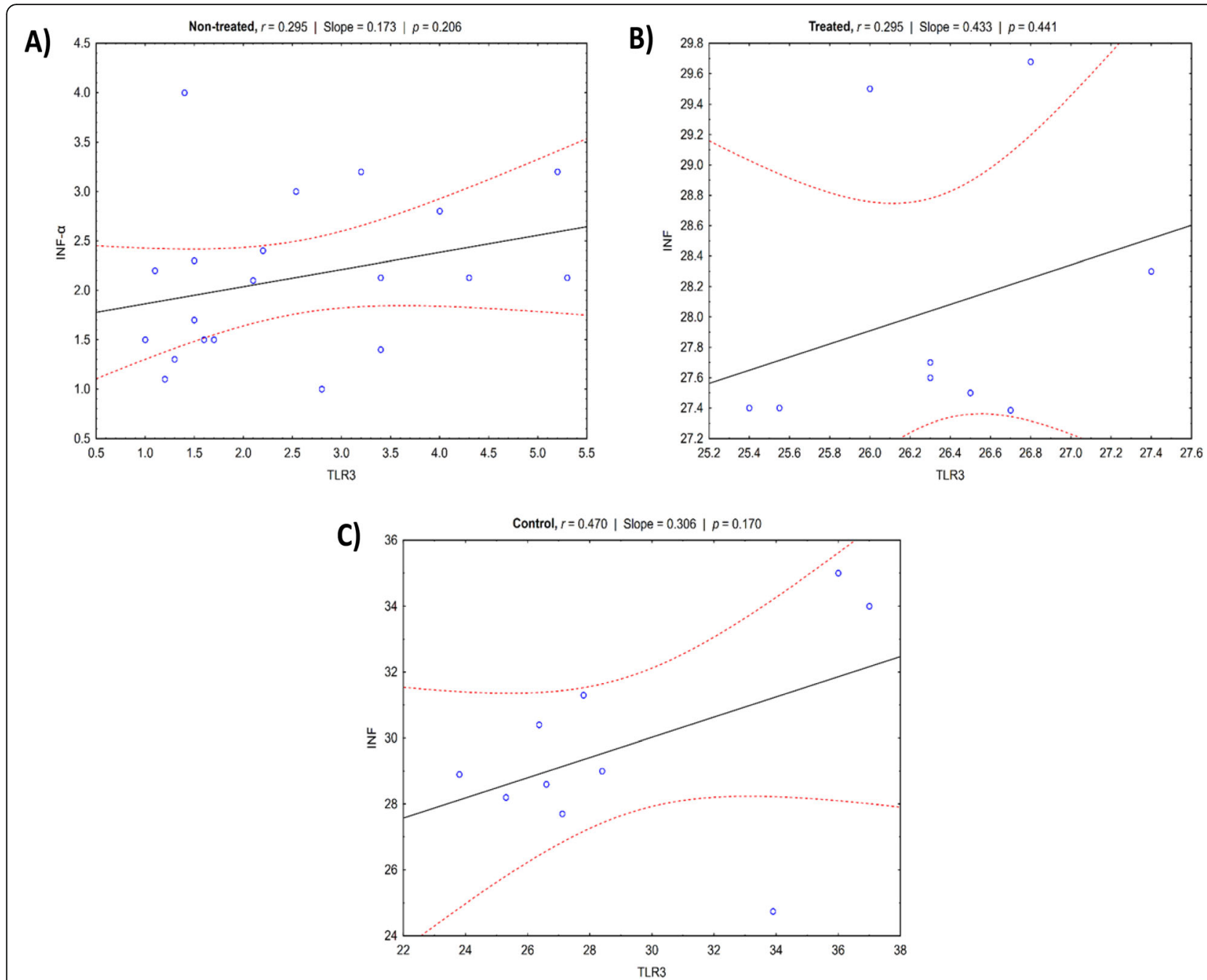

Fig. 4 a The correlation between expression levels of TLR3 and IFN-a in non-treated chronic HCV patients. $\mathbf{b}$ The correlation between expression levels of TLR3 and IFN-a in treated HCV group. $\mathbf{c}$ The correlation between expression levels of TLR3 and IFN-a in the control group

that coordinates adaptive immune responses to remove pathogens [16]. About HCV, one of the strategies used detects the presence of the virus by hepatocytes and innate immune cells is the triggering activation of TLR3, 7,8 , and 9 which are specific in HCV nucleic acids recognition. TLR3 bonded by dsRNA while TLR7/8 is recognizing ssRNA [17].

Upon engagement, these receptors initiating a cascade of events that results in transcription factor activation and induction of IFN-I and inflammatory cytokines leading to antiviral state acquisition [18]. It has been suggested that $\mathrm{HCV}$ uses several strategies to efficiently evade the IFN-I response through interfering with PRRs signaling cascade intermediate either by blocking TLR3mediated pathway by using serine protease NS3/4A to cleave the key adaptor of TLR3 [19], TRIF [20], or by induction of miR-21 that has been reported to suppress the TLR7-mediated pathway upon suppression of MyD88 and IRAK1 expression [2, 12, 21-23].

However, most of these reports have been developed on in vitro transfected cell cultures or animal experimental systems. Here, the objective of the presented study was to evaluate TLR3 and TLR7 expression in patients chronically infected with hepatitis $C$ virus infection and its association with IFN- $\alpha$ production. Our results indicated that the expression levels of TLR3, TLR7, and IFN- $\alpha$ were significantly downregulated in non-treated chronic HCV patients compared to both treated HCV patients and control subjects. On the other hand, treated $\mathrm{HCV}$ patients showed non-significant downregulation of the same three sensing receptors (TLR3, TLR7, and IFN- $\alpha$ ) compared to the control group. The expression levels of IFN- $\alpha$ were positively correlated with the levels of both TLR3 and TLR7. 

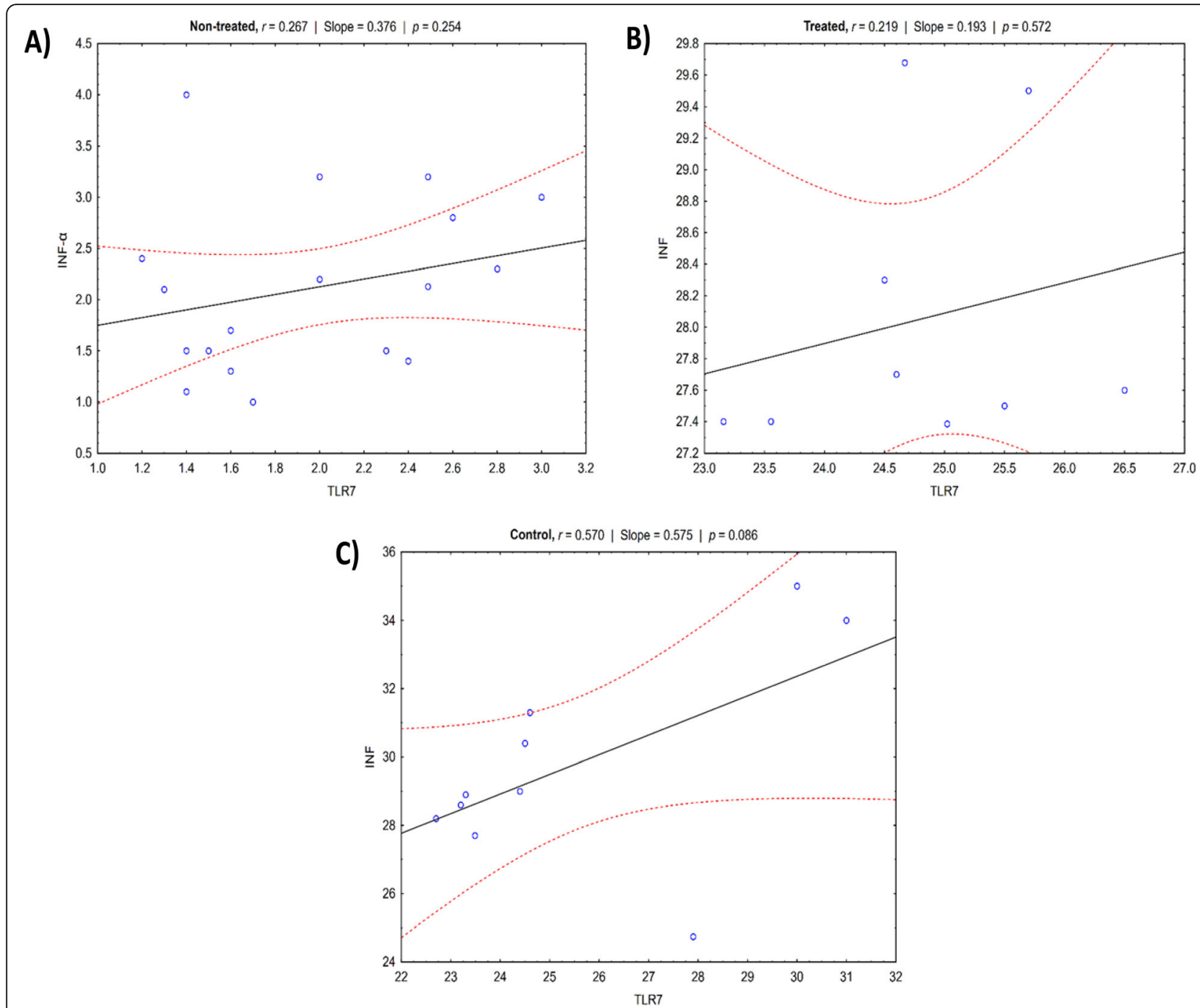

Fig. 5 a The correlation between expression levels of TLR7 and IFN-a in non-treated chronic HCV patients. $\mathbf{b}$ The correlation between expression levels of TLR7 and IFN- $a$ in treated HCV group. c The correlation between expression levels of TLR7 and IFN-a in the control group

Moreover, this downregulation in chronic HCV infection, could be one of the mechanisms used by HCV to interfere with an early innate immune response through impairment of the IFNs mediated antiviral mechanisms that may therefore lead to impaired $\mathrm{T}$ cell activation, in agreements with previous studies that have reported in mice showing lacking MyD88 as a result of TLR7 signal disturbance [24] that is responsible for the persistent replication and eventually promote disease progression and establishment, a chronic infection in $70-80 \%$ of infected patients $[19,25]$.

Several studies have examined the nature of the relationship between $\mathrm{HCV}$ infection and the gene expression rate of TLR 3, 7, 8, and 9. Our findings were consistent with other studies reported that the expression levels of TLR3 and TLR7 were downregulated in patients with chronic $\mathrm{HCV}$ infection when compared with healthy subjects $[26,27]$. Another study showed the decrease of TLR3 expression in the presence of HCV infection via NS4B-induced downregulating TRIF protein level also supported our findings [28]. Previous study examined a potential association between single-nucleotide polymorphisms (SNPs) in the TLR3, TLR9 genes, and HCV infection among Egyptian patients and showed its downregulation [29]. The presented study was also in agreement with reports that showed the expression levels TLR7 were significantly lowered in patients with chronic HCV and HCC compared to patients who naturally cleared their infection and controls [30].

However, our findings are contrary with another study that found HCV infection could lead to increased expression level of TLR7 mRNA in peripheral blood cells of HCV-infected samples [31]. However, this inconsistency can be due to the difference in patient's selection, 


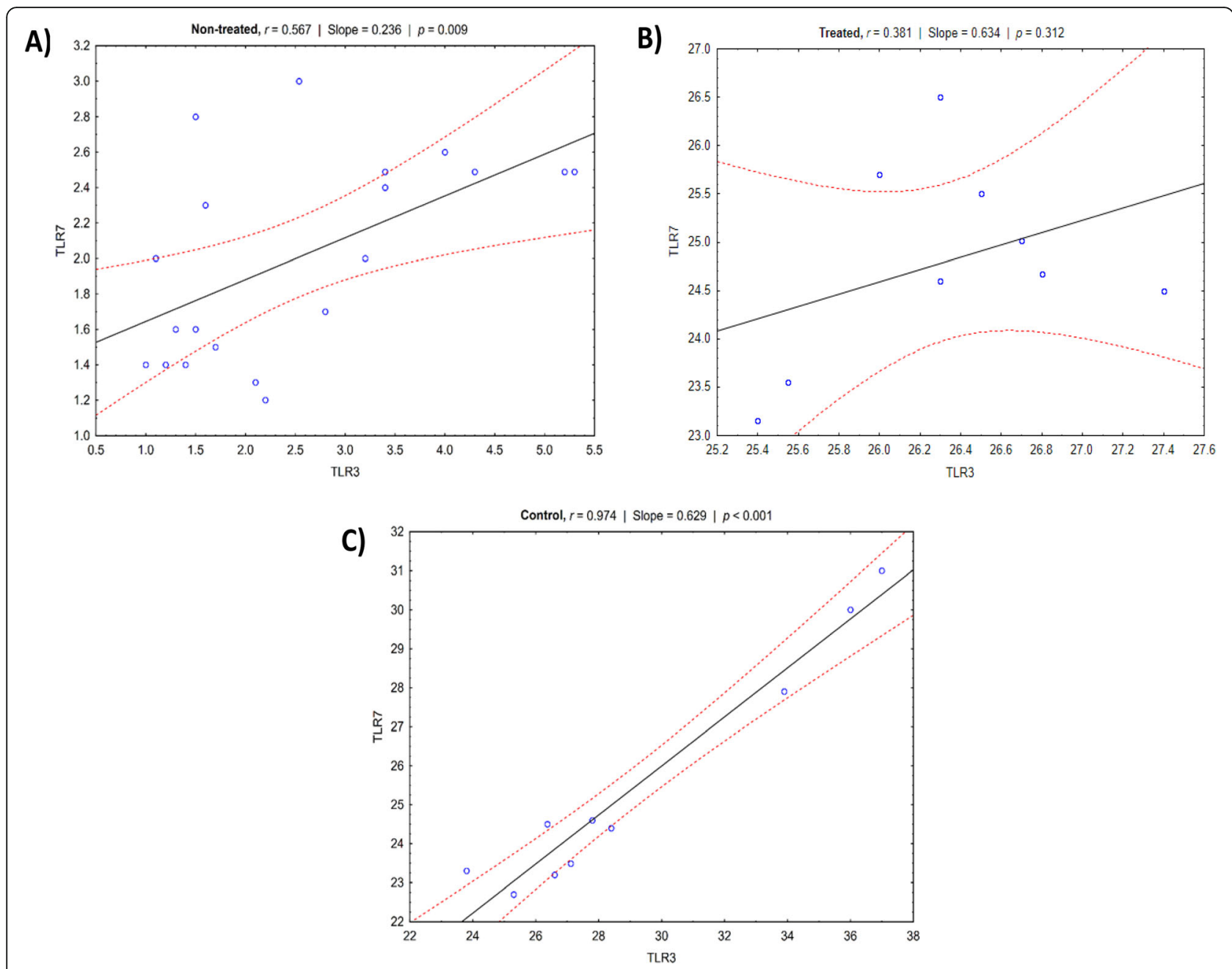

Fig. 6 a The correlation between expression levels of TLR3 and TLR7 in non-treated chronic HCV patients. $\mathbf{b}$ The correlation between expression levels of TLR3 and TLR7 in treated HCV group. $\mathbf{c}$ The correlation between expression levels of TLR3 and TLR7 in the control group

Table 1 The correlation among TLR3, TLR7, IFN-a, and laboratory and biochemical parameter findings in the chronic HCV patient

\begin{tabular}{|c|c|c|c|c|c|c|c|}
\hline & & ALT (IU/ML) & AST (IU/ML) & HCV PCR & WBCs (mg/dl) & $\mathrm{HB}(\mathrm{mg} / \mathrm{dl})$ & PLT (mg/dl) \\
\hline \multirow[t]{3}{*}{ TLR3 } & $r$ & 0.0367 & 0.0438 & 0.609 & 0.169 & 0.169 & 0.02 \\
\hline & $p$ value & 0.85 & 0.821 & 0.001 & 0.387 & 0.38 & 0.909 \\
\hline & & NS & NS & $S^{*}$ & NS & NS & NS \\
\hline \multirow[t]{3}{*}{ TLR7 } & r & 0.045 & 0.0809 & 0.377 & 0.201 & 0.0582 & 0.2 \\
\hline & $p$ value & 0.817 & 0.676 & 0.004 & 0.296 & 0.764 & 0.298 \\
\hline & & NS & NS & $S^{*}$ & NS & NS & NS \\
\hline \multirow[t]{3}{*}{ IFN- $a$} & $r$ & 0.0025 & 0.103 & 0.595 & 0.195 & 0.26 & 0.0521 \\
\hline & $p$ value & 0.99 & 0.595 & 0.001 & 0.31 & 0.173 & 0.788 \\
\hline & & NS & NS & $S^{*}$ & NS & NS & NS \\
\hline
\end{tabular}


methodological approaches, clinical stage, the genetic background of the population, and HCV genotypes. In agreement with other previous studies, the correlation among TLR3, TLR7, IFN- $\alpha$, and all biochemical parameters findings (ALT, AST, WBCs, HB, PLT) in the nontreated chronic HCV patients were non-significant [12]. We and other studies confirmed that the expression levels of TLR3 and TLR7 were strongly correlated with the expression level of IFN- $\alpha$ [27].

As a key component of innate immunity, the IFN system forms are the first line of defense against several pathogens to remove incoming infection and direct a subsequent adaptive response. From this standpoint, $\mathrm{HCV}$ infection leads to reduction in expression of TLRs (TLR3 and TLR7) on innate immune cells and hepatocytes with subsequent disruption of the process of IFN- $\alpha$ production which may be considered as an evolving strategy that allows the virus to exploit the immune system of its host and ensure survival and replication. So, the presented were designed to highlight a deeper understanding of the anti-immune mechanisms of the $\mathrm{HCV}$ that targets TLR signaling pathways, especially in a chronic stage, where HCV key protein players and regulators can be identified as useful targets. Also, the weaknesses of the host defenses can be detected and $\mathrm{HCV}$ immune response is more accurately controlled. Accordingly, several TLR ligands have been studied as a synthetic activator of certain TLRs. For instance, application of the TLR7 agonist ANA773 isatoribine has been shown to markedly enhance viral clearance by the immune system, as monitored by dose-dependent dynamics of immunological biomarkers [24]. In addition, synthetic dsRNA (poly I:C) TLR3 antagonists, such as dsRNA mimic polyinosinic: polycytidylic acid (poly I:C) induced stimulation and reactivation of DCs that could induce the immune responses against $\mathrm{HCV}$ infection [32].

\section{Conclusions}

We revealed that the expression levels of TLR3, TLR7, and IFN- $\alpha$ were significantly downregulated in nontreated chronic HCV patients compared to both treated $\mathrm{HCV}$ patients and control subjects. Also, the expression levels of IFN- $\alpha$ were positively correlated with the levels of both TLR3 and TLR7. These findings are an important issue for future research to detect the possible role of TLRs as a predictor of response to antiviral therapy are needed. Also, the possibility of targeting these receptors to enhance the immune response either nonspecific responses or $\mathrm{HCV}$-specific immunity to viral proteins in patients with other stages of disease needs further studies. It is important to bear in mind that even this study was conducted on only twenty non-treated chronic $\mathrm{HCV}$ patients and ten treated $\mathrm{HCV}$ patients, but further research and studies are hence needed to evaluate the effects on a larger number of cases will need to be undertaken. The studying of the crucial role of signal transduction via TLR3 and TLR7 in the proper activation of DCs and T cell priming specific to $\mathrm{HCV}$ is also needed to develop a full picture of successful immune response against $\mathrm{HCV}$.

\begin{abstract}
Abbreviations
DC: Dendritic cells; HCC: Hepatocellular carcinoma; CT: Cycle threshold; dsRNA: Double-stranded RNA; GAPDH: Glyceraldehyde 3 phosphate dehydrogenase; HCC: Hepatocellular carcinoma; HCV: Hepatitis C virus; IFNa: Interferon alpha; IRF3: Interferon regulatory factor 3; MyD88: Myeloid differentiation primary response 88; NF-kB: Nuclear factor NF-kappa-B; PAMPs: Pathogen-associated molecular pattern; PBMCs: Peripheral blood mononuclear cells; PRRs: Pathogen recognition receptor; RTqPCR: Quantitative real-time PCR; ssRNA: Single-stranded RNA; TLR3: Toll-like receptor3; TLR7: Toll-like receptor 7; TRIF: TIR-domain-containing adapterinducing interferon- $\beta$
\end{abstract}

\section{Acknowledgements}

We thank Dr. Nashwa Shebl for her kind help and all staff of the Virology and Molecular Biology unit at Children's Cancer Hospital Egypt-57357 (CCHE). Authors are greatly thankful to Dr. Albaraa El-Saied and Dr. Ehab Fathy for their support and assistance with the statistical analysis of this work.

\section{Authors' contributions}

MMSF created the study concept; MMSF, EEMM, MTMM, and KW contributed to experiments design; RAS, MTMM, and MMSF performed the experiments; MMSF and RAS drafted and revised the manuscript and performed the analysis of the data; MTMM, EEMM, and KW critically revised the manuscript. All authors gave final approval of the version to be published, and agree to be accountable for all aspects of the work.

\section{Funding}

Not applicable

\section{Availability of data and materials}

The datasets used and analyzed in the current study are available from the corresponding author upon reasonable request.

\section{Ethics approval and consent to participate}

All participants were consented to use their samples and clinical data (CHC patients) for this study. Informed written consent was obtained from each patient included in the study. The study protocol was approved by the Ethics Committee of the National Liver Institute, and the research was conducted in accordance with the principles of the Declaration of Helsinki; the committee's reference number is not available. We would like to confirm that this material is the authors' own original work, which has not been previously published elsewhere. The paper is not currently being considered for publication elsewhere. The paper reflects the authors' own research and analysis in a truthful and complete manner. The paper properly credits the meaningful contributions of co-authors and co-researchers. The results are appropriately placed in the context of prior and existing research. All authors have been personally and actively involved in substantive work leading to the manuscript and will hold themselves jointly and individually responsible for its content.

\section{Consent for publication}

Not applicable

\section{Competing interests}

The authors declare no conflict of interest.

\section{Author details}

${ }^{1}$ Botany and Microbiology Department, Faculty of Science, Al-Azhar University, 11884 Nasr City, Cairo, Egypt. ${ }^{2}$ Virology and Immunology Department, National Cancer Institute, Cairo University and Children Cancer Hospital Egypt, 57357 Cairo, Egypt. ${ }^{3}$ Department of Gastroenterology, 
Endocrinology, Rheumatology, and Infectious Diseases, University Hospital Regensburg, 93053 Regensburg, Germany.

Received: 14 August 2020 Accepted: 5 January 2021

Published online: 26 February 2021

\section{References}

1. Ray, Stuart C, Thomas et al (2009) "Chapter 154: Hepatitis C". Principles and practice of infectious diseases, 7th edn. Churchill Livingstone, Philadelphia ISBN 978-0-443-06839-3

2. Rosadini CV, Kagan JC (2015) Microbial strategies for antagonizing toll-likereceptor signal transduction. Curr Opin Immunol 32:61-70

3. Elgharably A, Gomaa Al, Crossey MM et al (2017) Hepatitis C in Egypt - past, present, and future. Int J Gen Med 10:1-6. https://doi.org/10.2147/IJGM. S119301

4. Kamal SM (2008) Acute hepatitis C: a systematic review. Am J Gastroenterol 103:1283e1297. https://doi.org/10.1111/j.1572-0241.2008.01825.x

5. Agrawal B, Krishnadas DK, Kumar R et al (2012) Proteins derived from hepatitis $C$ virus modulate immune responses: targeting dendritic cells. Int Immunol 22:27-48. https://doi.org/10.1093/intimm/dxq033

6. Saha B, Szabo G (2014) Innate immune cell networking in hepatitis C virus infection. J Leukoc Biol 96.5:757-766. https://doi.org/10.1189/Jlb.4MR0314$141 \mathrm{R}$

7. Santoro MG, Rossi A, Amici C (2003) NF-kB and virus infection: who controls whom. EMBO J 22:2552-2560. https://doi.org/10.1093/emboj/cdg267

8. Takeda K, Akira S (2004) TLR signaling pathways. Semin Immunol 16:3-9. https://doi.org/10.1016/j.smim.2003.10.003

9. Takeda K, Akira S (2005) Toll-like receptors in innate immunity. Int Immunol 17:1-14. https://doi.org/10.1093/intimm/dxh186

10. Hornung V, Rothenfusser S, Britsch S et al (2002) Quantitative expression of toll-like receptor 1-10 mRNA in cellular subsets of human peripheral blood mononuclear cells and sensitivity to CpG oligodeoxynucleotides. J Immunol 168:4531-4537. https://doi.org/10.4049/jimmunol.168.9.4531

11. Jacobs BL, Langland JO (1996) When two strands are better than one: the mediators and modulators of the cellular responses to double-stranded RNA. Virology. 219:339-349. https://doi.org/10.1006/viro.1996.0259

12. Baxt LA, Garza-Mayers AC, Goldberg MB (2013) Bacterial subversion of host innate immune pathways. Science 340:697-701

13. Matsumoto M, Kikkawa S, Kohase M et al (2002) Establishment of a monoclonal antibody against human toll-like receptor 3 that blocks doublestranded RNA-mediated signaling. Biochem Biophys Res Commun 293(5): 1364-1369. https://doi.org/10.1016/S0006-291X(02)00380-7

14. Chen K, Huang J, Liu Y et al (2009) Synergy of TRIF-dependent TLR3 and MyD88-dependent TLR7 in up-regulating expression of mouse FPR2, a promiscuous G-protein- coupled receptor, in microglial cells. J Neuroimmunol 213(1-2):69-77. https://doi.org/10.1016/j.jneuroim.2009.05. 018

15. Hiscott J, Nguyen TL, Arguello M et al (2006) Manipulation of the nuclear factor-kappa B pathway and the innate immune response by viruses. Oncogene. 25:6844-6867. https://doi.org/10.1038/sj.onc.1209941

16. Easterbrook PJ, Group WGD (2016) Who to test and how to test for chronic hepatitis C infection-2016 WHO testing guidance for low-and middleincome countries. J Hepatol 65(1):S46-S66

17. Pandey S, Kawai T, Akira S (2015) Microbial sensing by toll-like receptors and intracellular nucleic acid sensors. Cold Spring Harb Perspect Biol 7:a016246

18. Honda K, Taniguchi T (2006) IRFs: master regulators of signaling by toll-like receptors and cytosolic pattern-recognition receptors. Nat Rev Immunol 6: 644-658

19. Horner SM (2014) Activation and evasion of antiviral innate immunity by hepatitis C virus. J Mol Biol 426:1198-1209

20. Li K, Foy E, Ferreon JC et al (2005) Immune evasion by hepatitis C virus NS3/4A protease-mediated cleavage of the toll-like receptor 3 adaptor protein TRIF Proc. Natl Acad Sci USA 102:2992-2997

21. Chen Y, Chen J, Wang H et al (2013) HCV-induced miR-2 contributes to evasion of host immune system by targeting MyD88 and IRAK1. PLoS Pathog 9:e1003248

22. Versteeg GA, Garcia-Sastre A (2010) Viral tricks to grid-lock the type interferon system. Curr Opin Microbiol 13:508-516

23. Orzalli MH, Knipe DM (2014) Cellular sensing of viral DNA and viral evasion mechanisms. Annu Rev Microbiol 68:477-492
24. Urbanowicz, Zagożdżon, Ciszek M (2019) Modulation of the immune system in chronic hepatitis $\mathrm{C}$ and during antiviral interferon-free therapy. Arch Immunol Ther Exp 67(2):79-88). Birkhauser Verlag AG. https://doi.org/10. 1007/s00005-018-0532-8

25. Metz P, Reuter A, Bender S et al (2013) Interferon-stimulated genes, and their role in controlling hepatitis C. virus. J Hepatol 59:1331-1341

26. Motavaf M, Noorbakhsh F, Alavian SM et al (2014) Distinct toll-like receptor 3 and 7 expression in peripheral blood mononuclear cells from patients with chronic hepatitis C infection. Hepat Mon 14(4):e16421. https://doi.org/ 10.5812/hepatmon.16421

27. Mohammed Kl, Adel LA, Ali-Eldin FA et al (2013) Expression of toll like receptors 3 \& 7 in peripheral blood from patients with chronic hepatitis $C$ virus infection and their correlation with interferon-alpha. Egypt J Immunol 20(1):13-22

28. Liang, Cao, Ding et al (2018) Hepatitis C virus NS4B induces the degradation of TRIF to inhibit TLR3-mediated interferon signaling pathway. PLoS Pathog 14(5):e1007075. https://doi.org/10.1371/journal.ppat.1007075

29. Hamdy, Osman, Zakaria et al (2018) Association of toll-like receptor 3 and toll-like receptor 9 single-nucleotide polymorphisms with hepatitis $C$ virus persistence among Egyptians. Arch Virol 163(9):2433-2442. https://doi.org/ 10.1007/s00705-018-3893-8

30. Ghonaim, Aziz, Mostafa et al (2019) Toll-like receptor 7 expression and gene polymorphism in patients with hepatitis $C$ and hepatocellular carcinoma. Egypt J Med Microbiol 28:4

31. Dehghan-Manshadi M, Hadinedoushan H, Amirbaigy MK et al (2015) Relative expression of toll-like receptors 2 and $7 \mathrm{mRNA}$ in peripheral blood of patients with hepatitis C. Hepat Mon 15(11):e30427. https://doi.org/10. 5812/hepatmon.30427

32. Salem M, Ibrahim EM, El-Bate $\mathrm{H}$ et al (2018) Ex vivo generation and maturation of dendritic cells from peripheral blood mononuclear cells of patients with chronic HCV or hepatocellular carcinoma using toll-like receptor 3 ligand poly(l:C). Int J Cancer Biomed Res 2(1):11-18. https://doi. org/10.21608/jcbr.2019.34740

\section{Publisher's Note}

Springer Nature remains neutral with regard to jurisdictional claims in published maps and institutional affiliations.

\section{Submit your manuscript to a SpringerOpen ${ }^{\circ}$ journal and benefit from:}

- Convenient online submission

- Rigorous peer review

- Open access: articles freely available online

High visibility within the field

- Retaining the copyright to your article

Submit your next manuscript at $>$ springeropen.com 